Tetrahedron Letters

journal homepage: www.elsevier.com

\title{
Bismuth(III) triflate catalyzed tandem esterification-Fries-oxa-Michael route to 4-chromanones
}

\author{
Kevin Meraz, Krishna Kumar Gnanasekaran, Rup Thing ${ }^{\dagger}$ and Richard A. Bunce* \\ Department of Chemistry, Oklahoma State University, Stillwater, OK 74078-3071, USA
}

\section{ARTICLE INFO}

\section{Article history:}

Received

Received in revised form

Accepted

Available online

\section{Keywords:}

4-chromanones

bismuth(III)triflate catalysis

tandem reaction

esterification-Fries-oxa-Michael reaction

\section{ABSTRACT}

An efficient tandem reaction approach is described to prepare 4-chromanones from electron-rich phenols and 3,3-dimethylacrylic acid or trans-crotonic acid in boiling toluene using $20 \mathrm{~mol} \%$ bismuth(III) triflate as the catalyst. The reaction is also successful from the corresponding aryl esters of each of these acids under the same conditions. The procedure is convenient to perform, and 25 $90 \%$ yields of products are realized following chromatography. A range of substrates is included (14 substrates for each acid) to help define the scope of the process. Additional experiments are reported, which confirm that the sequence of events involves (1) esterification, (2) Fries rearrangement and (3) oxa-Michael ring closure.

2009 Elsevier Ltd. All rights reserved.

The development of efficient methods to produce oxygen heterocycles for use as building blocks in natural product and drug synthesis is a worthy endeavor. Among the heterocyclic scaffolds containing oxygen, 4-chromanones are widely dispersed in nature and constitute valuable substrates for drug synthesis. ${ }^{1,2}$ For example, 4-chromanones are core structures in a family of anti-estrogenic compounds used to treat breast cancer. ${ }^{3}$ Various derivatives of this system are also inhibitors of SIRT2, an enzyme involved in agerelated neurodegenerative disorders. ${ }^{4}$ Finally, 4-chromanones have been used in the synthesis of somatostatin analogues which are currently under investigation as a possible treatment for osteoporosis $^{5}$ as well as certain stomach tumors. ${ }^{6}$

We have previously reported synthetic routes to tetrahydronaphthalenes, $^{7}$ tetrahydroisoquinolines $^{8}$ and tetrahydroquinolines ${ }^{9}$ using relatively mild catalysts for ring closure via a Friedel-Crafts alkylation. We have also communicated an Amberlyst-15 promoted regioselective addition of $\alpha, \beta$-unsaturated ketones to the para position of phenols. ${ }^{10}$ In the current report, we wish to disclose our use of bismuth(III) triflate for the one-pot synthesis of 4chromanones by a tandem esterification-Fries rearrangementintramolecular oxa-Michael addition sequence. Our approach involves the reaction of electron-rich phenols with 3,3dimethylacrylic acid or trans-crotonic acid to give di-, tri- and tetrasubstituted 4-chromanones.

Previous methods for the synthesis of 4-chromanones have utilized a wide range of reaction protocols. One of the first reports

\footnotetext{
*Corresponding author. Tel.: +1 405744 5952; fax: +1 4057446007.

E-mail address: rab@okstate.edu (R.A. Bunce).

† Summer 2016 NSF-REU student from Southeastern Oklahoma State University, Durant, OK 74701, USA.
}

described the preparation of 7-hydroxy-2,2-dimethyl-4-chromanone from resorcinol and 3,3-dimethylacrylic acid in the presence of antimony(III) chloride. $^{11}$ Since then, the amine-catalyzed condensation of $o$-hydroxyacetophenones with aliphatic ketones and aldehydes has proven to be a more versatile approach. ${ }^{3-6,12-14}$ Further accounts have detailed the use of stoichiometric mercury(II) on aryl propargyl ethers, ${ }^{15}$ the addition of cuprates to chromones, ${ }^{16}$ the treatment of phenols and 3,3-dimethylacrylic acid with excess phosphorus oxychloride/zinc chloride, ${ }^{17,}{ }^{18}$ the reaction of carboxylic acids with $o$-(trimethylsilyl)aryl triflates promoted by cesium fluoride $^{19}$ and a one-pot, base-mediated photo-Fries-oxa-Michael reaction. ${ }^{20}$

The current project originated from efforts to extend the FriedelCrafts ring closure of phenyl 3,3-dimethylacrylate $(\mathbf{1 a}, \mathrm{X}=\mathrm{H})$ to other phenol-derived acrylate esters. Cyclization of $\mathbf{1 a}$ is reported to give 4,4-dimethyl-2-chromanone (2a) in $>70 \%$ yield using 1.7 equiv of aluminum chloride in dichloromethane, ${ }^{21}$ and we have successfully employed this procedure to access drug precursors for one of our projects. Attempts to perform this reaction with esters incorporating more activated aryl groups, however, led to mixtures of several products. For example, 1c $(\mathrm{X}=\mathrm{Me})$ produced 1-(2hydroxy-4-methylphenyl)-3-methyl-2-buten-1-one (3c, 18\%) and 2,2,7-trimethyl-4-chromanone $(\mathbf{4 c}, 65 \%)$ in addition to a small amount of $2 \mathbf{c}(2 \%)$ (see Scheme 1). The major 4-chromanone product, in this case, likely arose via a Lewis acid promoted Fries rearrangement of $\mathbf{1 c}$ to give $\mathbf{3 c}$, followed by a 6-endo-trig oxaMichael ring closure. ${ }^{22}$ While this may appear to be a reasonable synthesis of 4-chromanones, the use of excess aluminum chloride for this conversion invariably led to mixtures of 2,3 and 4 . Thus, we sought a more selective route to 4 -chromanone derivatives. 
We have previously described the use of bismuth(III) triflate as a catalyst for Friedel-Crafts cyclizations of tertiary alcohols to produce chromans as well as a variety of other heterocycles. ${ }^{23}$ As an extension of this earlier work, trial experiments using bismuth(III) triflate with ester 1c revealed that these substrates afforded $>70 \%$ of $\mathbf{4 c}$ as the exclusive cyclized product, along with $5-15 \%$ of phenol $\mathbf{5 c}$ derived from Lewis acid catalyzed cleavage of the ester. Additional examples showed that this process appeared to be broadly applicable to many substrates (see Table 1). Other triflate salts, including those of magnesium, scandium(III), copper(II), zinc and silver(I), as well

Scheme 1. Friedel-Crafts reaction of aryl esters of 3,3-dimethylacrylic acid.

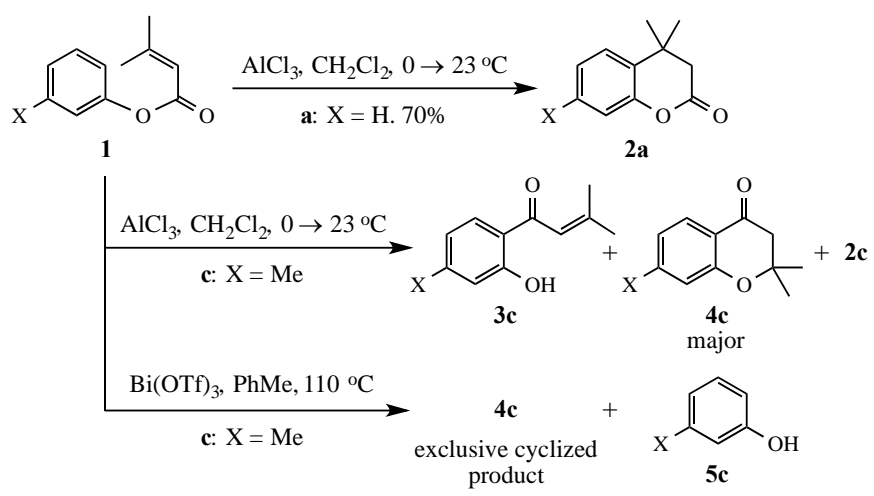

as indium(III) chloride and Amberlyst-15, proved far less effective as catalysts for this conversion with most affording yields significantly below $50 \%$. Optimization studies further indicated that $20 \mathrm{~mol} \%$ of bismuth(III) triflate gave the best results. Attempts to

Table 1. 4-Chromanones from aryl esters of 3,3-dimethylacrylic acid.

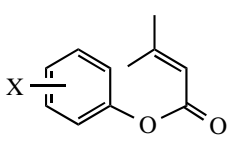

1

Entry

${ }^{\text {a }}$ Starting esters were prepared as described in ref 21.

${ }^{\mathrm{b}}$ The product was accompanied by small quantities of the corresponding phenol. ${ }^{\mathrm{c}}$ The product accompanied by $12 \%$ of the starting ester and $30 \%$ of the phenol. use additional catalyst, as might be required for an acylation process, not only proved expensive and operationally difficult (with a MW = 656 , an additional $10 \mathrm{~mol} \%$ represents a significant quantity), but also gave lower yields and made purification more difficult.

To avoid the extra step of preparing esters 1 from the relatively expensive of 3,3-dimethylacryloyl chloride, we sought to prepare 4chromanones directly from phenols and 3,3-dimethylacrylic acid using bismuth(III) triflate. Fortunately, this route proved viable and gave fair to excellent yields of 4-chromanones with none of the 2chromanone products. In no case were Fries-derived 1-(2hydroxyaryl) enones isolated from the bismuth(III)-catalyzed reactions. Additionally, there was no migration of alkyl groups in the substrates or participation by the toluene solvent during the transformation. Among the activated substrates studied, 2methylphenol was the lone disappointment, giving an inseparable mixture of products from which the 4-chromanone could not be readily isolated in pure form. We were also successful in expanding this protocol to cyclizations with trans-crotonic acid. However, attempts to further extend this process to ring closures of transcinnamic acid gave only intractable mixtures with poor yields of the desired products.

The results of our heterocyclization studies of phenols 5 with 3,3dimethylacrylic acid (6) and crotonic acid (7) are summarized in Tables 2 and 3, respectively. Each reaction was run in toluene at 110 ${ }^{\circ} \mathrm{C}$ using a $1: 1$ ratio of phenol to carboxylic acid and $20 \mathrm{~mol} \%$ of bismuth(III) triflate. The mixtures were refluxed for $12-24 \mathrm{~h}$ and monitored by TLC. When maximum conversions were attained, the reactions were cooled and concentrated under vacuum. The crude products were then applied directly to preparative thin layer chromatography plates and eluted several times with $5 \%$ ether in hexane (8-10\% ether in hexane for the methoxy-substituted products) to afford three major bands. In each case, the bright fluorescent blue band was the 4-chromanone, while other bands contained the aryl ester of the corresponding acid ( $\mathbf{1}$ from acid $\mathbf{6}$ or $\mathbf{9}$ from acid $\mathbf{7}$ ) and the starting phenol.

The reaction using acid $\mathbf{6}$ proceeded smoothly for a broad range of substrates and the resulting 4-chromanones were easily purified by chromatography. Good yields were achieved with most of the alkylsubstituted phenols (entries b-d, i-k and m). Lower yields, however, were noted for 2,5-dialkylphenols (entries $\mathbf{e}$ and $\mathbf{h}$ ), which are sterically hindered and also tend to sublime into the reflux condenser. Efforts to circumvent the sublimation problem by performing the reaction of $\mathbf{5 e}$ with $\mathbf{6}$ under sealed tube conditions failed to improve the yield, suggesting that the major problem in these cases was steric in nature. For monocyclic phenols bearing substitution at $\mathrm{C} 3$ relative to the $\mathrm{OH}$ (entries $\mathbf{c}, \mathbf{f}, \mathbf{g}, \mathbf{i}$, and $\mathbf{n}$ ), the Fries rearrangement occurred regioselectively away from the substituted position. More complex phenols such as 2-naphthol (entry l) and 5,6,7,8-tetrahydro-2-naphthol (entry m) proceeded in high yield, but interestingly gave different regioselectivities in the Fries rearrangement. For 2-naphthol, the acyl group migrated to $\mathrm{C} 1$ (toward the fused ring) to generate an angular tricyclic structure after ring closure, while the tetrahydro derivative underwent acyl migration to $\mathrm{C} 3$ (away from the fused ring) leading to a linear tricyclic system. As expected, lower yields were observed with 3chlorophenol (entry $\mathbf{n}$ ), demonstrating the effect of a deactivating substituent. The analogous 3-fluorophenol (entry l), however, was unique in having an electron-withdrawing group while still giving a good yield of product. This is typical of monofluoroaromatics, which often show high reactivity in the para position relative to the fluorine. ${ }^{24,25}$ 
Table 2. 4-Chromanones from 3,3-dimethylacrylic acid (6).

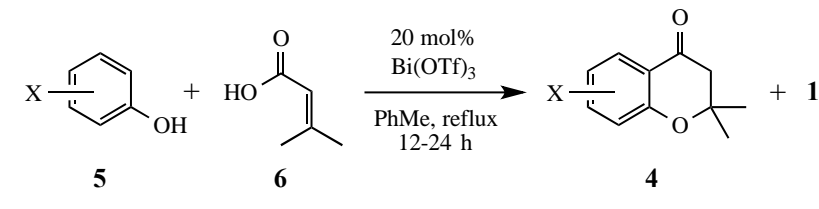

Entry

${ }^{\text {a }}$ The product was accompanied by $5-15 \%$ of the corresponding phenol.

${ }^{\mathrm{b}}$ The aryl ester $\mathbf{1}$ of acid $\mathbf{6}$ was isolated for entries $\mathbf{e}, \mathbf{h}$ and $\mathbf{n}$ (see the SI) in addition to $5-15 \%$ of the corresponding phenols.

Thus, for 3-fluorophenol, the fluorine activates the para position that accepts the acylium ion during the Fries rearrangement, and the outcome was better than expected. Lastly, the two methoxysubstituted substrates (entries $\mathbf{g}$ and $\mathbf{o}$ ) gave moderate yields of cyclized products, with the 3-methoxy derivative giving the lower yield (for an improvement, see below). The yields of 4-chromanones
Table 3. 4-Chromanones from trans-crotonic acid (7).

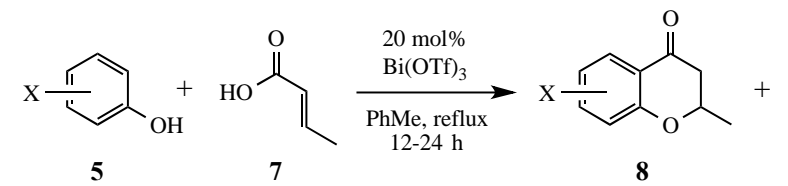

Entry

${ }^{a}$ The product was accompanied by $5-15 \%$ of the corresponding phenol.

${ }^{b}$ The aryl ester $\mathbf{9}$ of acid $\mathbf{7}$ was isolated for entries $\mathbf{e}, \mathbf{h}, \mathbf{j}$, and $\mathbf{n}$ (see the SI) in addition to $5-15 \%$ of the corresponding phenols.

8 from crotonic acid (7) were, for the most part, comparable to those from 3,3-dimethylacrylic acid (6), despite the reduced partial positive charge at the enone terminus in the bismuth-coordinated Fries product.

In general, the yields of $\mathbf{4}$ from aryl 3,3-dimethylacrylates $\mathbf{1}$ were similar to those from $\mathbf{5}$ and $\mathbf{6}$ since the ester proved to be the first 
intermediate in the sequence (see Scheme 2). The higher yield obtained from the 3-methoxyphenyl ester $\mathbf{1 g}$ (versus the reaction of $\mathbf{5 g}$ with 6) is notable, however, and shows the potential effect of water on acid-sensitive substrates. When starting from the acid, water generated in the esterification process, and not immediately removed by the boiling toluene, could potentially react with the catalyst to form traces of triflic acid sufficient to cleave the methoxy ether and lower the yield. Starting from the ester, however, water would not be produced and the reaction might be expected to give a higher yield of the desired product. Finally, to rule out water as a problem in the sealed tube reactions of 2,5-dialkylphenols, ester $1 \mathbf{e}$ was heated in a sealed tube with $20 \mathrm{~mol} \%$ of bismuth(III) triflate in toluene at $120{ }^{\circ} \mathrm{C}$ for $24 \mathrm{~h}$. By this route, the yield of chromanone $4 \mathrm{e}$ was essentially the same as that achieved from phenol and the acid, which supports our supposition that steric factors were largely responsible for the low yields from these substrates.

A plausible mechanism for the conversion of $\mathbf{5 c}$ and $\mathbf{6}$ to $\mathbf{4 c}$ is shown in Scheme 2. Close monitoring of the process by thin layer chromatography indicated that the first intermediate was the aryl ester 1c. While this produces an equivalent of water, which could decompose the catalyst, the boiling toluene solvent should remove most of this product from the reaction by azeotropic distillation into the reflux condenser, thus minimizing catalyst degradation. The ester would then undergo a Fries rearrangement via $\mathbf{1 0}$ and $\mathbf{1 1}$ to give 1(2-hydroxy-4-methylphenyl)-3-methyl-2-buten-1-one (3c). While
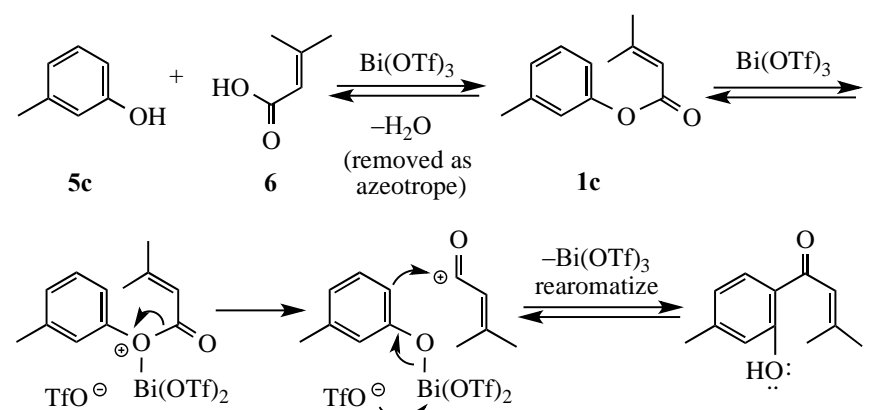

10 11
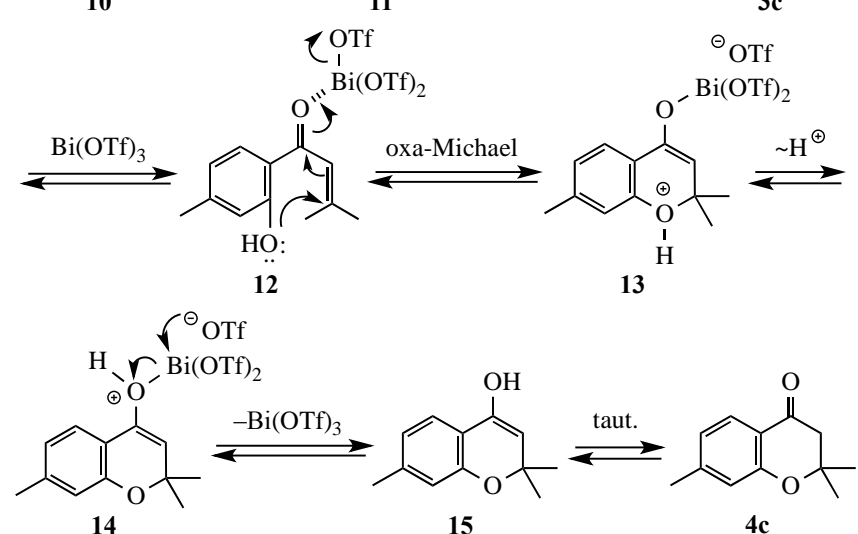

Scheme 2. A plausible mechanism for the $\mathrm{Bi}(\mathrm{OTf})_{3}$ catalyzed synthesis of 4chromanones.

this compound proved to be a significant product in the reaction promoted by excess aluminum chloride, a control experiment demonstrated that bismuth(III) triflate rapidly and quantitatively converted 3c, presumably via intermediates $\mathbf{1 2 - 1 5}$, to the ring-closed product $\mathbf{4 c}$. The failure of $\mathbf{3 c}$ to completely cyclize under the aluminum chloride conditions likely resulted from strong coordination of both the phenol and side chain ketone functions by the excess Lewis acid. This coordination would diminish the nucleophilicity of the ortho hydroxyl group and slow the oxaMichael addition to the enone double bond. Once ring closure of $\mathbf{1 2}$ to $\mathbf{1 3}$ occurs in the bismuth(III) catalyzed reaction, proton transfer to generate 14, disengagement of the catalyst to give enol 15, and tautomerization would lead to the final product.

In conclusion, we have described an efficient bismuth(III) catalyzed tandem reaction to prepare 4-chromanones from electronrich phenols and 3,3-dimethylacrylic acid or trans-crotonic acid. The procedure is convenient to perform, product purification is straightforward, and the target heterocycles are isolated in fair to excellent yields. A reasonable selection of substrates has been surveyed to define the scope of the reaction. Limitations are predictably associated with deactivating groups on the aromatic nucleus and steric hindrance toward reattachment of the acyl group to the aromatic ring during the Fries rearrangement. Additionally, experiments have confirmed that the sequence of events during the reaction involves (1) esterification of the acid by the phenol, (2) Fries rearrangement of the 2-butenyl acylium fragment to the less hindered ortho position and (3) oxa-Michael ring closure of the phenolic $\mathrm{OH}$ to the side chain enone of the Fries product. We are continuing our work to identify mild catalytic processes for the synthesis of heterocyclic compounds of interest to natural product and drug research.

\section{Acknowledgments}

R.T. wishes to thank the 2016 REU Program at Oklahoma State University (NSF CHE-1559874) for a summer appointment. The authors also wish to acknowledge the NSF (BIR-9512269), the Oklahoma State Regents for Higher Education, the W. M. Keck Foundation, and Conoco, Inc. for funding to establish the Oklahoma Statewide Shared NMR Laboratory. The College of Arts and Sciences at OSU is also acknowledged for funds to purchase a new $400 \mathrm{MHz}$ NMR for this facility.

\section{Supplemental information}

Supplementary information associated with this article (experimental procedures and spectral data) can be found, in the online version, at http://dx.doi.org/10.1016/j.tetlet

\section{References}

1. Timar, T.; Levai, A.; Eszenyi, T.; Sebok, P. J. Heterocycl. Chem. 2000, 37, 1389-1417.

2. Emami, S.; Ghanbarimasir, Z. Eur. J. Med. Chem. 2015, 93, 539563.

3. Zhu, M.; Kim, M. H.; Lee, S.; Bae, S. J.; Kim, S. H.; Park, S. B. J. Med. Chem. 2010, 53, 8760-8764.

4. Friden-Saxin, M.; Seifert, T.; Landergren, M. R.; Suuronen, T.; Lahtela-Kakkonen, M.; Jarho, E. M.; Luthman, K. J. Med. Chem. 2012, 55, 7104-7113.

5. Draper, R. W.; Hu, B.; Iyer, R. V.; Li, X.; Lu, Y.; Rahman, M.; Vater, E. J. Tetrahedron 2000, 56, 1811-1817.

6. Friden-Saxin, M.; Seifert, T.; Malo, M.; Andersson, K. d. S.; Pemberton, N.; Dyrager, C.; Friberg, A.; Dahlen, K.; Wallen, E. A. A.; Groetli, M.; Luthman, K. Eur. J. Med. Chem. 2016, 114, 59-64.

7. Bunce, R. A.; Cox, A. N. Org. Prep. Proced. Int. 2010, 42, 83-93.

8. Bunce, R. A.; Cain, N. R.; Cooper, J. G. Org. Prep. Proced. Int. 2012, 44, 131-145.

9. Bunce, R. A.; Cain, N. R.; Cooper, J. G. Org. Prep. Proced. Int. 2013, 45, 28-43. 
10. Bunce, R. A.; Reeves, H. D. Synth. Commun. 1989, 19, 1109-1117.

11. Miyano, M.; Matsui, M. Bull. Chem. Soc. Jpn. 1958, 31, 397-402.

12. Banerji, A.; Goomer, N. C. Tetrahedron Lett. 1979, 20, 3685-3686.

13. Shawcross, F.; Sard, H. J. Heterocycl. Chem. 1995, 32, 1393-1395.

14. Park, S. O.; Kim, J.; Koh, M.; Park, S. B. J. Comb. Chem. 2009, 11, 315-326.

15. Gopalsamy, A.; Balasubramanian, K. K. J. Chem. Soc., Chem. Commun. 1988, 34-35.

16. Saengchantara, S. T.; Wallace, T. W. Tetrahedron 1990, 46, 30293036.

17. Timar, T.; Jaszberenyi, J. C. J. Heterocycl. Chem. 1988, 25, 871877.

18. Sebok, P.; Jeko, J.; Timar, T.; Jaszberenyi, J. C. Tetrahedron Lett. 1992, 33, 2791-2794.

19. Dubrovskiy, A. V.; Larock, R. C. Tetrahedron 2013, 69, 27892798.

20. Iguchi, D.; Erra-Balsells, R.; Bonesi, S. M. Tetrahedron Lett. 2014, 55, 4653-4656.

21. Dawson, M. I.; Hobbs, P. D.; Derdzinski, K.; Chan, R. L. S.; Gruber, J.; Chao, W.; Smith, S.; Thies, R. W.; Schiff, L. J. J. Med. Chem. 1984, 27, 1516-1531.

22. Baldwin, J. E.; Thomas, R. C.; Kruse, L. I.; Silberman, L. J. Org. Chem. 1977, 42, 3846-3852.

23. Nammalwar, B.; Bunce, R. A. Tetrahedron Lett. 2013, 54, 43304332.

24. Ault, A. J. Chem. Educ. 1966, 43, 329-330.

25. Rosenthal, J.; Schuster, D. I. J. Chem. Educ. 2003, 80, 679-690. 


\section{Graphical Abstract}

\section{Bismuth(III) triflate catalyzed esterification-Fries-}

oxa-Michael route to 4-chromanones

Leave this area blank for abstract info.

Kevin Meraz, Krishna Kumar Gnanasekaran, Rup Thing and Richard A. Bunce*

Department of Chemistry, Oklahoma State University, Stillwater, OK 74078-3071, USA

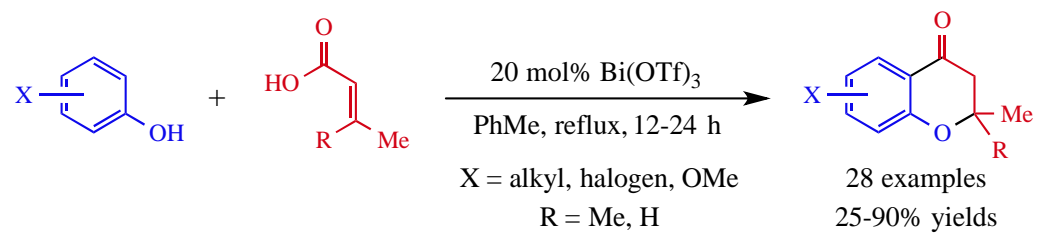

